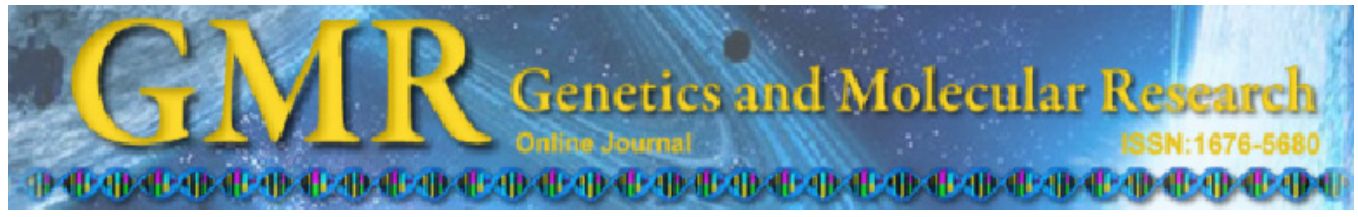

Short Communication

\title{
Polymorphic microsatellite loci for the Japanese anchovy Engraulis japonicus (Engraulidae)
}

\author{
L. Lin ${ }^{1,2}$, L. Zhu' ${ }^{2}$ S.-F. Liu' ${ }^{2}$ Y.-Q. Su${ }^{1}$ and Z.-M. Zhuang ${ }^{2}$ \\ ${ }^{1}$ College of Oceanography and Environmental Science, Xiamen University, \\ Xiamen, China \\ ${ }^{2}$ Key Laboratory for Fishery Resources and Eco-environment, \\ Shandong Province, Yellow Sea Fisheries Research Institute, \\ Chinese Academy of Fishery Sciences, Qingdao, China \\ Corresponding author: Z.-M. Zhuang \\ E-mail: zhuangzm@ysfri.ac.cn
}

Genet. Mol. Res. 10 (1): 764-768 (2011)

Received October 1, 2010

Accepted November 24, 2010

Published May 3, 2011

DOI 10.4238/vol10-2gmr1085

\begin{abstract}
We isolated and characterized 14 polymorphic microsatellite loci in the Japanese anchovy (Engraulis japonicus) using a $(\mathrm{GT})_{13}$-enriched genomic library. The numbers of alleles per locus ranged from 6 to 31 , with a mean of 17.8. The observed and expected heterozygosities ranged from 0.180 to 0.949 and from 0.172 to 0.966 , with means of 0.731 and 0.825 , respectively. All 14 loci were in Hardy-Weinberg equilibrium and no significant linkage disequilibrium between loci pairs was detected. These microsatellite markers will be useful for analyzing the population genetic structure and gene flow of E. japonicus.
\end{abstract}

Key words: Engraulis japonicus; Japanese anchovy;

Microsatellite loci; Population structure 


\section{INTRODUCTION}

The Engraulis japonicus anchovy is a small pelagic, schooling fish widely distributed in the northwest Pacific from southern Sakhalin Island, Russia to Guangdong, China (Liu et al., 2006). In the 1990s the anchovy fishery in China was a major part of the China's marine fishing industry with the largest annual landing of 1.37 million tons in 1998 (Jin et al., 2001). The E. japonicus anchovy is also regarded as an ecologically key species because it is an important food source for high-valued fish such as the Spanish mackerel, small yellow croaker and tailfish. However, the anchovy stock in China has drastically declined over the last decade mainly due to overexploitation and environmental changes (Jin et al., 2001). Characterizing the population structure of E. japonicus may lead to a better understanding of the effect on overexploitation and environmental changes and give new insights into maintenance and efficient management of anchovy resources.

Pelagic marine fishes generally show low levels of genetic differentiation among geographic regions due to the lack of physical barriers to genetic exchange in "open" oceans and their biological characteristics such as large population size and relatively long planktonic early life stages (Liu et al., 2006; Zhan et al., 2009). Among molecular markers, microsatellite markers are seen as the best way to identify population structure in pelagic marine fishes because of their hypervariability, abundance, neutrality, codominance, and unambiguous scoring of alleles (Tautz, 1989; Zhan et al., 2009). Previously, six polymorphic microsatellite loci were isolated to examine the population structure of two geographic populations in Taiwan (Chiu et al., 2002; Yu et al., 2002). Here we describe the development of an additional 14 loci that will increase the power available for detecting fine-scale population genetic structure and gene flow of E. japonicus.

\section{MATERIAL AND METHODS}

Forty individuals were collected from Qingdao, China. Samples were preserved at $-20^{\circ} \mathrm{C}$ until DNA extraction. A dinucleotide-enriched genomic library was constructed following Ma's method (Ma and Chen, 2009). In brief, genomic DNA was extracted from muscle tissue and digested with MseI restriction enzyme (New England Biolabs, USA). The DNA fragments were ligated to the adapters (5'-TAC TCA GGA CTC AT-3'/5'-GAC GAT GAG TCC TGA G-3'). Then the ligated products were pre-amplified in a $25-\mu \mathrm{L}$ reaction system using the adapter specific primer (5'-GAT GAG TCC TGA GTA A-3') to verify successful ligation and increase DNA concentration. Bio-labeled probes $(\mathrm{GT})_{13}$ were applied to hybridize with the products from pre-amplification. Subsequently, the hybrids were captured by the streptavidin-coated magnetic beads (Promega, USA), and the obtained DNA fragments eluted from the magnetic beads were amplified by the adapter specific primer. The final amplification products were ligated into the pMD 18-T vectors (TaKaRa, Japan) and transformed into Escherichia coli DH5 $\alpha$ competent cells. The positive clones were randomly sequenced by ABI Prism 3730 automated DNA sequencer (Applied Biosystems, USA). Microsatellite repeats were found in 282 of sequenced clones. Polymerase chain reaction (PCR) primer pairs were designed to amplify 149 microsatellite loci with suitable flanking regions using the PRIMER PREMIER 5 software (Premier Biosoft International, USA). 
The designed primers were evaluated by 40 individuals of E. japonicus. PCR was performed on a Veriti Thermal Cycler (Applied Biosystems) in a total volume of 25 $\mu \mathrm{L}$ concluding $0.4 \mu \mathrm{M}$ each primer, $0.2 \mathrm{mM}$ each dNTP, $1 \mathrm{X}$ PCR buffer, $2 \mathrm{mM} \mathrm{MgCl}$, 1 unit Taq polymerase (Fermentas, USA) and 10-100 ng DNA. The PCR cycling profile consisted of one cycle at $94^{\circ} \mathrm{C}$ for $5 \mathrm{~min}, 35$ cycles of $45 \mathrm{~s}$ at $94^{\circ} \mathrm{C}, 1 \mathrm{~min}$ at the locusspecific annealing temperature (Table 1), $45 \mathrm{~s}$ at $72^{\circ} \mathrm{C}$, and a final cycle of $10 \mathrm{~min}$ at $72^{\circ} \mathrm{C}$. The PCR products were separated on $6 \%$ denaturing polyacrylamide gel, and visualized by silver staining. The size of allele was estimated according to the pBR322/ MspI marker (TianGen, China). The observed and expected heterozygosities together with tests for Hardy-Weinberg equilibrium and linkage disequilibrium were calculated by GENEPOP 4.0 (Rousset, 2008). Null allele frequencies (Brookfield, 1996) were calculated by MICRO-CHECKER 2.2.3 (Van Oosterhout et al., 2004). All results for multiple tests were corrected using Bonferroni's correction (Rice, 1989).

\section{RESULTS AND DISCUSSION}

Forty-two loci were reliably amplified and showed polymorphism. The observed genotypes deviated from Hardy-Weinberg expectations in 27 loci after Bonferroni's correction ( $\mathrm{P}$ $<0.0012$ ), resulting from heterozygote deficiency. Null alleles may be present in 17 loci (null allele frequency $>10 \%$ ). As a result, there were 14 loci without deviation from Hardy-Weinberg equilibrium or high frequencies of null alleles. Among these 14 reliable loci, the numbers of alleles per locus ranged from 6 to 31, with an average of 17.8. The observed and expected heterozygosities ranged from 0.180 to 0.949 and from 0.172 to 0.966 , with an average of 0.731 and 0.825 , respectively (Table 1 ).

Twenty-seven of 42 loci deviated from Hardy-Weinberg equilibrium in this study. Similar results were reported in the previous microsatellite marker developments of $E$. japonicus and E. encrasicolus, where 5 of 6 and 3 of 5 loci deviated significantly from HardyWeinberg equilibrium, respectively (Chiu et al., 2002; Landi et al., 2005). As analyzed, there are three possible causes for the deviation from Hardy-Weinberg equilibrium: 1) high ratio of allele number to sample size, which is beyond the appropriate parameter space for these tests (Rubin et al., 2009); 2) the presence of null alleles (Chiu et al., 2002; Landi et al., 2005), and 3 ) the subpopulations existing in the sample (Wahlund effect) (Chiu et al., 2002; Landi et al., 2005). Nevertheless, in the microsatellite marker development of E. encrasicolus, Pakaki et al. (2009) reported that all 11 microsatellite loci were in Hardy-Weinberg equilibrium except for one locus in a test population and two loci in the other test population, which may be related to how the markers were developed. First, only those loci with fewer than 14 uninterrupted repeats were selected for primer design; second, only those primer pairs that performed well in genotyping were used to screen for polymorphism in the two test populations. Consequently, most loci deviating from Hardy-Weinberg equilibrium actually were ignored.

\section{ACKNOWLEDGMENTS}

Research supported by the National Natural Science Foundation of China (grant \#40776097 and \#31061160187) and the National High Technology Research and Development Program of China (grant \#2009AA09Z401). 


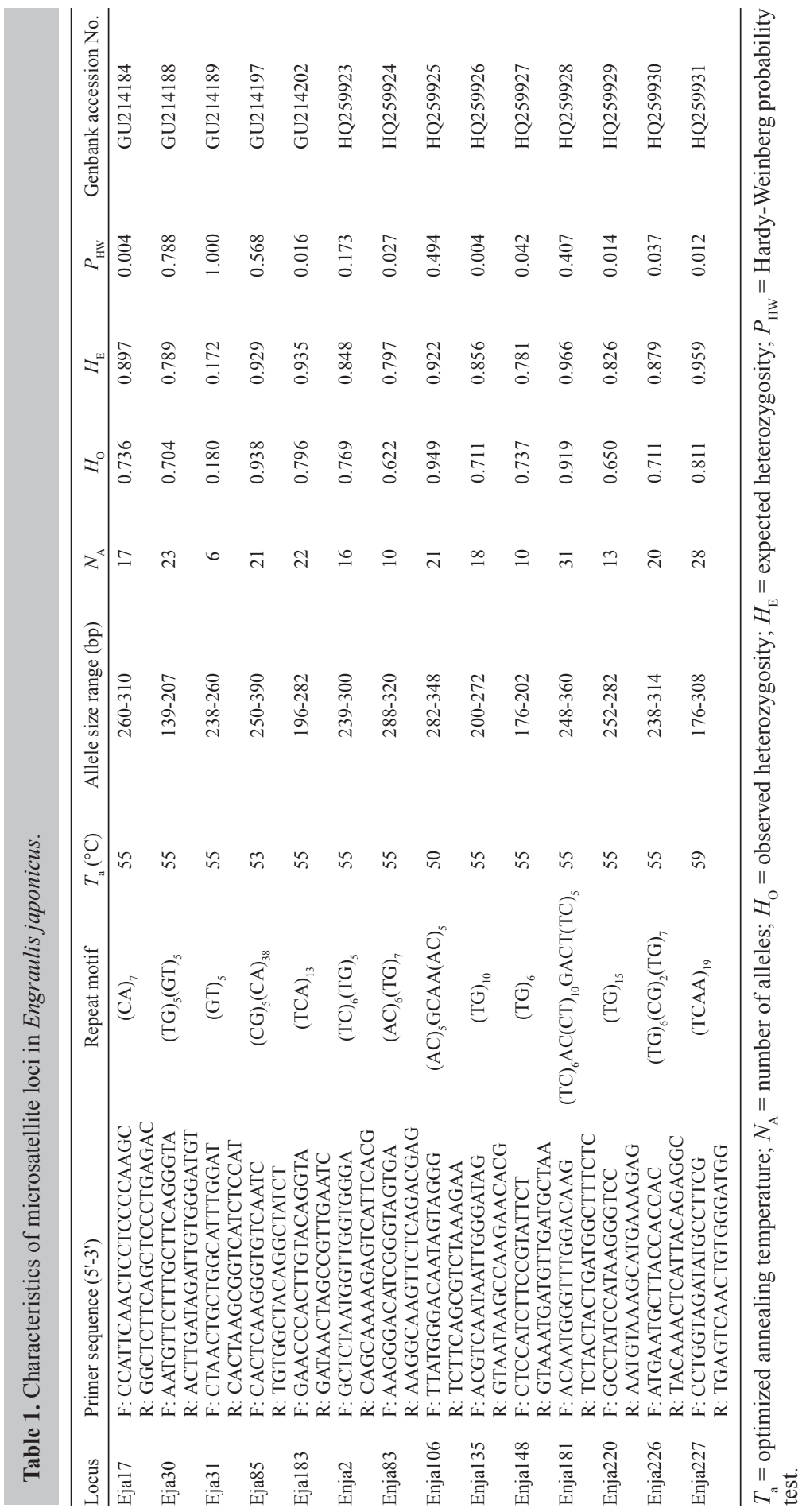




\section{REFERENCES}

Brookfield JF (1996). A simple new method for estimating null allele frequency from heterozygote deficiency. Mol. Ecol. 5: 453-455.

Chiu TS, Lee YJ, Huang SW and Yu HT (2002). Polymorphic microsatellite markers for stock identification in Japanese anchovy (Engraulis japonica). Mol. Ecol. Notes 2: 49-50.

Jin XS, Hamre J, Zhao XY and Li FG (2001). Study on the quota management of anchovy (Engraulis japonicus) in the Yellow Sea. J. Fish. Sci. China 8: 27-30.

Landi M, Garoia F, Piccinetti C and Tinti F (2005). Isolation of polymorphic microsatellite loci from the European anchovy, Engraulis encrasicolus. Mol. Ecol. Notes 5: 266-268.

Liu JX, Gao TX, Zhuang ZM, Jin XS, et al. (2006). Late Pleistocene divergence and subsequent population expansion of two closely related fish species, Japanese anchovy (Engraulis japonicus) and Australian anchovy (Engraulis australis). Mol. Phylogenet. Evol. 40: 712-723.

Ma HY and Chen SL (2009). Isolation and characterization of 31 polymorphic microsatellite markers in barfin flounder (Verasper moseri) and the cross-species amplification in spotted halibut (Verasper variegatus). Conserv. Genet. 10 : 1591-1595.

Pakaki V, Magoulas A and Kasapidis P (2009). New polymorphic microsatellite loci for population studies in the European anchovy, Engraulis encrasicolus (L.). Mol. Ecol. Notes 9: 1406-1409.

Rice WR (1989). Analyzing tables of statistical tests. Evolution 43: 223-225.

Rousset F (2008). GENEPOP '007: a complete re-implementation of the GENEPOP software for Windows and Linux. Mol. Ecol. Notes 8: 103-106.

Rubin BE, Makarewich CA, Talaba AL, Stenzler L, et al. (2009). Isolation and characterization of microsatellite markers from the acacia-ant Crematogaster mimosae. Mol. Ecol. Res. 9: 1212-1214.

Tautz D (1989). Hypervariability of simple sequences as a general source for polymorphic DNA markers. Nucleic Acids Res. 17: 6463-6471.

Van Oosterhout C, Hutchinson WF, Wills DPM and Shipley P (2004). MICRO-CHECKER: software for identifying and correcting genotyping errors in microsatellite data. Mol. Ecol. Notes 4: 535-538.

Yu HT, Lee YJ, Huang SW and Chiu TS (2002). Genetic analysis of the populations of Japanese anchovy (Engraulidae: Engraulis japonicus) using microsatellite DNA. Mar. Biotechnol. 4: 471-479.

Zhan A, Hu J, Hu X, Zhou Z, et al. (2009). Fine-scale population genetic structure of Zhikong scallop (Chlamys farreri): do local marine currents drive geographical differentiation? Mar. Biotechnol. 11: 223-235. 